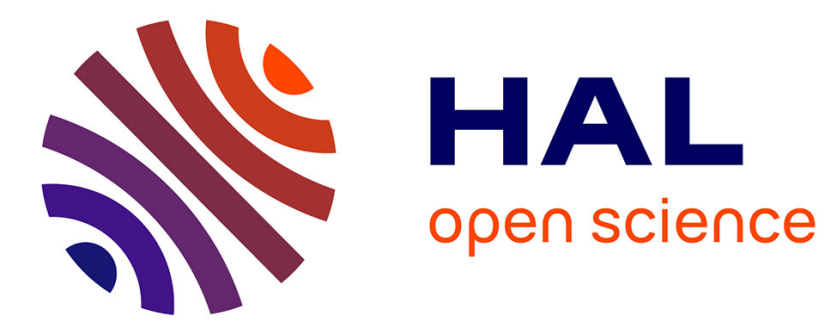

\title{
Les îles de Glénan et leurs abords
}

André Guilcher

\section{To cite this version:}

André Guilcher. Les îles de Glénan et leurs abords. Bulletin de l'Association de géographes français, 1937, 105, pp.72-79. 10.3406/bagf.1937.7013 . insu-02276842

\section{HAL Id: insu-02276842 \\ https://hal-insu.archives-ouvertes.fr/insu-02276842}

Submitted on 4 Mar 2021

HAL is a multi-disciplinary open access archive for the deposit and dissemination of scientific research documents, whether they are published or not. The documents may come from teaching and research institutions in France or abroad, or from public or private research centers.
L'archive ouverte pluridisciplinaire $\mathbf{H A L}$, est destinée au dépôt et à la diffusion de documents scientifiques de niveau recherche, publiés ou non, émanant des établissements d'enseignement et de recherche français ou étrangers, des laboratoires publics ou privés.

\section{()ㅜ) $\Theta$}

Distributed under a Creative Commons Attribution - NoDerivatives| 4.0 International 


\section{Les îles de Glénan et leurs abords}

\section{André Guilcher}

\section{Citer ce document / Cite this document :}

Guilcher André. Les îles de Glénan et leurs abords. In: Bulletin de l'Association de géographes français, N¹05, 14e année, avril 1937. pp. 72-79;

doi : https://doi.org/10.3406/bagf.1937.7013

https://www.persee.fr/doc/bagf_0004-5322_1937_num_14_105_7013

Fichier pdf généré le 25/04/2018 
Communication de M. A. Gurcher

\section{Les iles de Glénan et leurs abords}

I. L'archipel des Glénon (figure 1) : Situé au S.-W. de Penmarc'h, fermant au $S$. l'Anse de Bénodet, l'archipel des Glénan est un plateau granulitique d'environ 7 milles $1 / 2$ de long sur 4 de large. Allongé d'W. en $E$, il est à 8 inilles $1 / 2$ de la pointe de Trévignon et $\dot{a} 11$ milles de la pointe de Mousterlin. Ce plateau porte dans sa partie orientale un grand nombre d'iles et îlots: Penfret, Guiautec, Le Loc'h, Fort-Cigogne, Bananec, Brunec, St-Nicolas, Drenec, Quignenec, etc... Au contraire, la partie occidentale n'est occupée que par des récifs, et les fonds sont plus considérables.

a) Les petits ilots et les récifs. A part St-Nicolas, le Loc'h et Penfret, les îlots composant les Glénan sont très peu étendus. Drence a encore $1 / 4$ de mille de diamètre; mais l'ile Cigogne est occupée tout entière par le vieux fort de Vauban, pourtant peu considerable; quant aux trois îlots de Quignenec, à Brunec, a Guiautec, ce sont des terres minuscules ayant de $100 \dot{a}$ 50 mètres de diamètre.

Toutes ces terres sont très basses. Un des îlots de Quignenec n'est pas à 2 mètres au-dessus des plus hautes mers. Drenec atteint de 2 à 4 mètres; Brunec, de 4 à 5 mètres. C'est dire qu'elles sont essentiellement sous l'empire de la mer. Cependant celle-ci, dans les conditions actuelles, n'a pu les aplanir à ce niveau. Aussi est-on amené, avec une grande probabilité, a en faire des éléments de la plate-forme monastirienne, dont laltitude moyenne sur les cotes finistériennes est de $1 \mathrm{~m} .50$ à 5 mètres. Én plusieurs points, notamment à Brunec, une nette rupture de pente sépare cette plate-forme de la plate-forme d'abrasion actuelle. D'autre part, dans l'ìlot $E$. de Quignenec, on trouve, sur cette plate-forme nettement abrasée et située à +4 mètres, deux gros monadnocks très caractéristiques.

Enfin, plusieurs rochers de l'archipel sont nettement aplanis vers +3 à +5 mètres. Le niveau monastirien semble donc jouer un grand rôle dans la topographie de ces îlots.

b) Le Loc'h. Cette île est la plus large, sinon la plus longue des îles de Glènan : près de 1 mille du N.-N.-W. au S.-S.-E. sur 1/2 mille de l'W. à l'E., et, si l'on en excepte les points N.-N.-W. et S.-S.-I., clle est presque ronde. Elle est tout a fait intéressante par ce fail qu'on y trouve la plus nette et la plus étenduc de toutes les plate-formes monastiriennes que nous ayons vues dans les îles finistériennes. On voit cette plate-forme, parfaitement déblayée, au niveau constant de +4 à +5 mètres faire le tour 
de l'île à l'E., au S., à l'W., large parfois de 200 mètres. La roche s'y présente à nu, ou recouverte de quelques centimètres à peine done terre très sableuse. La plate-forme granulitique, par suite du clivage subhorizontal de la roche, se décompose en grandes dalles qui donnent à cette surface pourtant si plate un aspect chaotique. La rupture de pente entre ce niveau et la plate-forme actuelle est partout très nette, et l'attaque par la mer de la plate-forme monastirienne se manifeste par la cassure de cette dernière en énormes dalles inclinées vers la mer, d'un curieux effet.

Vers l'intérieur de l'île, la plate-forme disparâ̂t sous un anneau de sable peu épais, qui entoure un étang assez vaste mais peu profond reposant vraisemblablement sur la plate-

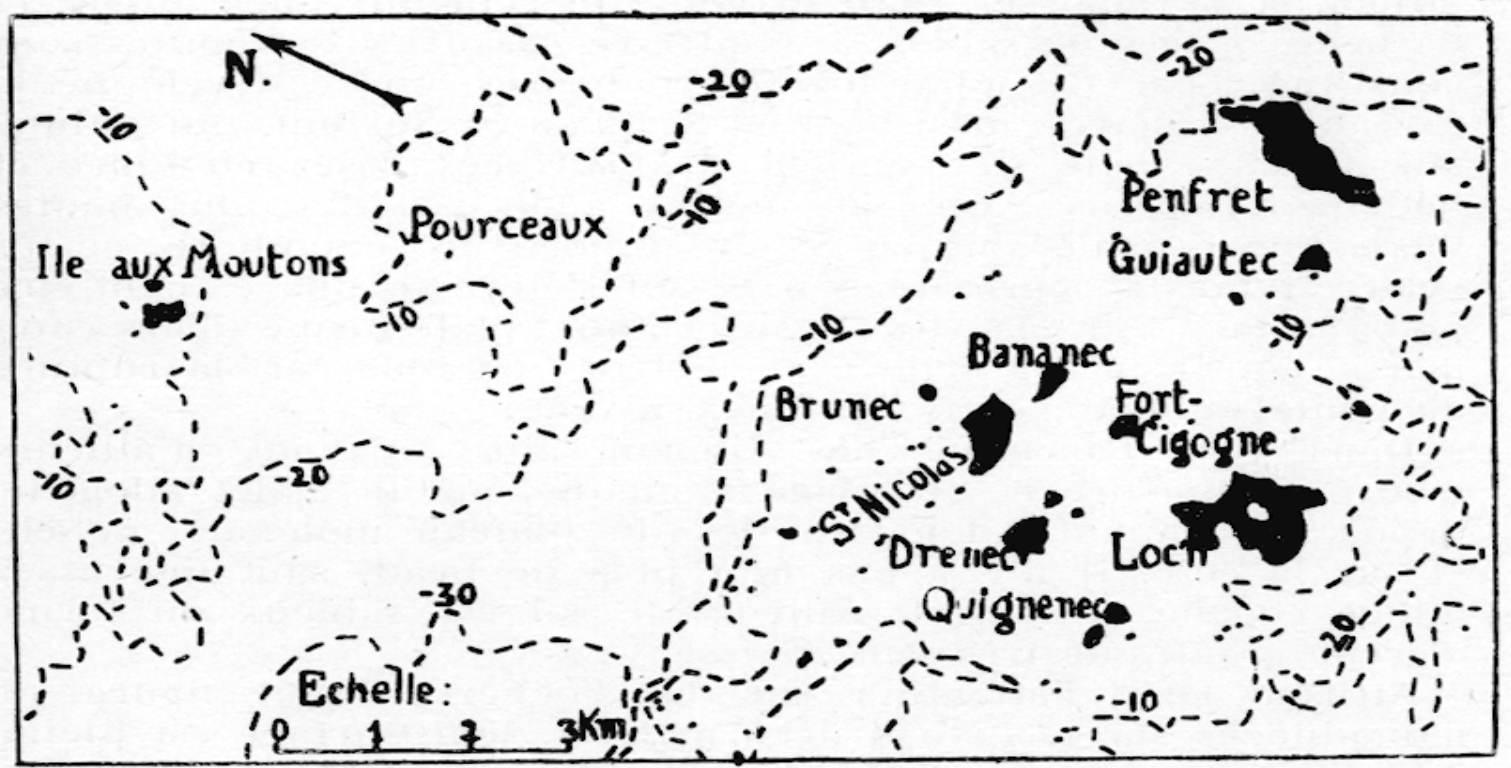

Fig. 1. - Les Glénan et lóle aux Moutons

- - isobathes

forme monastirienne. L'écoulement se fait vers le Sud, à la faveur d'une entaille dans la plate-forme, à travers du sable et de gros blocs de granulite. Enfin, tout le N. de l'île est occupé par des dunes.

L'extension considérable du niveau monastirien de 5 mètres est donc le caractère principal de l'île du Loc'h, ainsi nommée à cause de son étang. Remarquons d'ailleurs qu'on trouve de tels étangs dans plusieurs îles du plateau molénais.

c) Saint-Nicolas. Ici on retrouve encore le niveau de 5 mè- 
tres sur le rïvage $S$. de l'île, en continuité vraisemblable sous la dune avec un autre lambeau du même niveau sur la rive $E$. Mais l'originalité de cette île, assez petite (1/2 mille de long), réside dans l'abondance des formations dunaires et la beauté des cordons de sable, dessinant des anses très régulières. Un cordon part de l'îlot situé à l'W. de Saint-Nicolas, longe la rive N.-W. et s'avance vers Brunec. Un autre longe la rive N.-E., de Brunec à Bananec. L'ìle est donc entourée de hauts cordons qui, entre eux, laissent une dépression au milieu de l'île, sans que, toutefois, il s'y forme d'étang. La topographie de cette île est donc le résultat de phénomènes plus récents que la topographie générale du I,oc'h.

d) Penfret. Allongée dans le sens N.-S., c'est la plus longue des Glénan : 1 mille $1 / 4$ sur $1 / 4$ à $1 / 2$ mille. Elle s'étrangle au milieu, et s'y abaisse : c'est là que l'on rencontre des dunes, $\in$ de belles plages de sable. Au contraire, les deux extrémités sont constituées par des plate-formes rocheuses, où se développe le niveau de 5 mètres aussi bien au $S$. qu'au $N$. Surtout, fait unique aux Glénan, cette île contient des surfaces assez étendues et nettement abrasées vers 20 mètres au-dessus des plus hautes mers. Peu considérable au $S$., où il porte le sémaphore, ce niveau est mieux représenté au N. où s'élève le phare. Peut-être pourrait-on voir là les restes d'une plate-forme d'abrasion marine supérieure à celle de 5 mètres; en tous cas, la rupture de pente est nette entre les deux niveaux.

Dans tout l'archipel des Glénan, nous n'avons d'ailleurs trouvé aucune trace de plage ancienne, aucun galet allogène, dépòts si communs dans les iles du plateau molénais, à Sein et sur la côte. Il n'y a pas non plus de head, sauf une assez mince couche à Penfret. Ailleurs le sol est sableux ou formé l'arène granitique très peu épaisse.

Attirons aussi l'attention sur les rochers faisant figure de monadnocks sur le niveau de 5 mètres : leur surface est pleine de sortes de cuvettes réunies par des canaux parfaitement polis et arrondis : ces formes ne se rencontrent aussi nettes qu'à Penmarc'h et à Sein, régions également granulitiques.

e) L'ile aux Moutons. Cette île est en dehors des Glénan : clle se trouve à 5 milles au $N$. de Saint-Nicolas, et séparée de celle-ci par deux chenaux encadrant le plateau des Pourceaux. Malgré son extrême petitesse (à peine $1 / 4$ de mille de long, et un rétrécissement central en réduit la largeur à 100 mètres environ), cette île est aussi intéressante que les Glénan proprement dites, et même davantage à certains égards.

D'abord on $y$ trouve une plate-forme, montant depuis $+1 \mathrm{~m}$. 50 ou même $+1 \mathrm{~m}$. jusque vers +5 ou +6 . Vers l'intérieur, elle disparaît en général sous du sable ou une très mince couche de head. Fille est très nette surtout à l'W., où sa pente, 
de ja a $7^{\circ}$, recoupe les diaclases de la granulite qui ont 35 à $40^{\circ}$. près du phare, elle supporte de petits monadnocks très nettement sapés à la base, qui sont à environ $+2 \mathrm{~m}$.

Mais surtout on trouve sur cette plate-forme des galets anciens. Au: S. et au S.-F. existe une plage ancienne, fossilisant la plateforme, épaisse d'environ 50 centimètres, et contenant, a còté de quelques gros blocs arrondis de granulite, des galets allogènes. D'autre part, face au $\mathbf{N}$, on rencontre des galets enchàssés dans la base du head, et j'y ai trouvé un gros grès armoricain et mòme un galet de silex.

Enfin a l'E. de l'ile existe un ilot minuscule de 15 mètres de rayon environ, uni à lîle a basse mer, constitué d'une plateforme $\dot{a}+2 \mathrm{~m}$. environ, fossilisée par une belle plage ancienne de $0 \mathrm{~m}$. 50 d'épaisseur, ou les galets de granulite voisinent avec ceux de gres, de schiste, de micaschiste, de quartzite et de silex.

L'île aux Moutons est donce plus riche en dépôts de galets ancicns que les Glénan : d'ailleurs celles-ci sont également pauvres en grèves de galets actuels et souvent, des éboulis de gros blocs, on passe sans transition au sable fin.

II. Les fonds avoisinants (1) : Au N.-E. des Glénan s'étend une vaste dépression, parallèle à la cote, depuis l'entrée de Lorient jusqu'à l'anse de Bénodet. Vers le large, cette dépression, qui descend très doucement du N.-W. et du S.-F. jusque vers 50 mètres près de la bouée de la Basse-Jaune (au S.-S.-E. de la pointe de Trévignon), est limitée par une série de hauts fonds et d'îles : Groix, basses de Groix et du Caudan, basse Jaune, plateau des Glénan, île aux Moutons, basses Tudy. Ces hauts fonds dominent de façon assez abrupte la grande dépression : dénivellation brusque de 30 à 40 mètres sur le front $\mathbf{N}$.-E. du plateau de la Basse-Jatine; de 20 a 25 mètres sur le front $N$.-E. du plateau des (ilénan, et mìme de 35 à 40 mètres devant Penfret; de 15 mètres devant l'ile aux Moutons. Au contraire, ces plateaux sousmarins s'abaissent graduellement vers le S.-W. Penfret, la plus haute des Glénan, est au N.-E. de lärchipel; plus au S.-W. se trouvent successivement les iles aplanies de $+1 \dot{a}+j$ mètres, puis les rochers isolés, enfin les basses, de plus en plus profondes.

Ia grande dépression est parcourue par les thalwegs sousmarins au profil très doux, provenant du Blavet, de l'Isole, de la rivière de Belon, de l'Aveu, de l'anse de la Forêt, de l'Odet et de la rivière de Pont-l'Abbé. 'Tous convergent vers une sortie commune, située à l'E. de Ia Basse-Jaune.

Ces faits font penser à un affaissement du sol sur l'emplacement de cette vaste dépression, qui serait limitée vers le S.-W. par un abrupt de faille très démantelé, au delà duquel le sol

(1) Voir les cartes $5.285,5.301,5.304,5.405$ et surlout 5.368 . Se reporter i la tigure 2. 


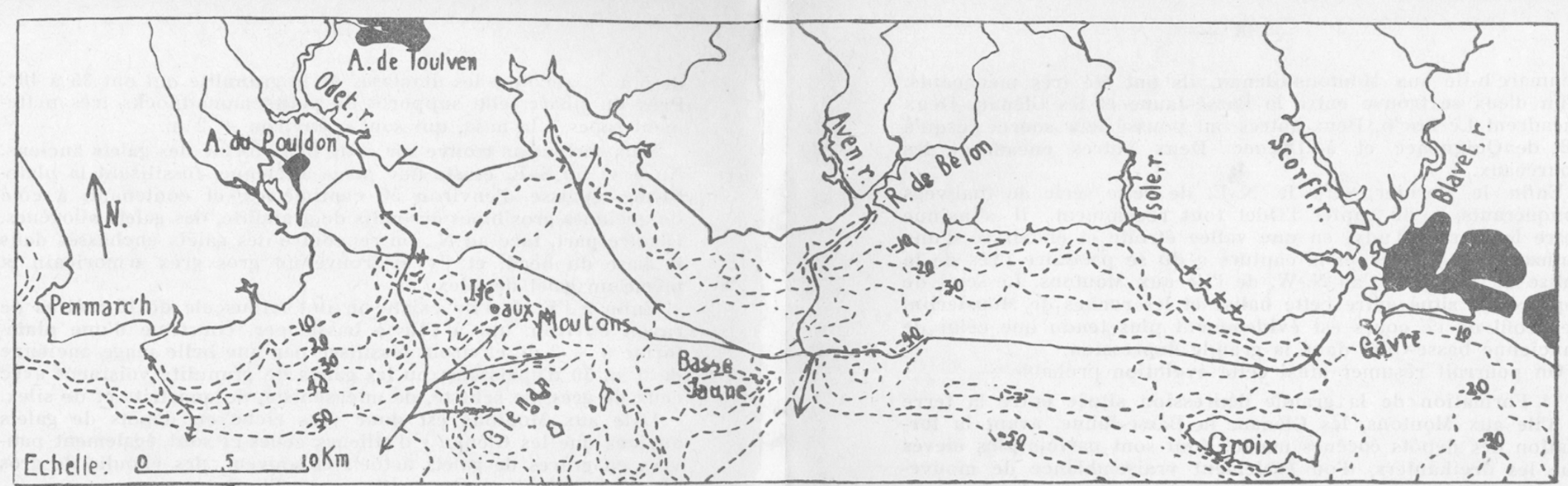

Fiti. 2. - Dépression. Les Griénan-Grotx

dreikanters
encène marin

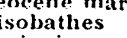

F principaux thalwegs sous-marins

descendrait dans l'ensemble en pente assez douce. Autrement dit, les Glénan et le plateau de la Basse-Jaune seraient un bloc basculé vers le S.-W.; sans en être sûr, on a quelques présomp tions de mouvements de ce genre sur d'autres points du littoral finisterien (1). L'abrupt de faille (ou de flexure) supposé s'atténue de plus en plus vers le N.-W.

Or, la ria de Quimper contient des dépôts marins éocènes (2) : grès analogues aux grès à sabals, calcaires, silex. On a trouve aussi, rejetes par la mer et provenant des fonds voisins, calcaires écènes marins sur le rivage de la prescu'te de Gâvre (3) ; près de lá, entre les estuaires du Blavet et de rivière d'Etel, affleurent des sables glauconieux à numites (4). On a aussi signalé, sur le rivage entre l'Isole et le Scorff des 4 dreikanters en grès éocenes contemporains des grès a Sabals (5). Enfin, nous avons pu determiner personnel

(1) Guiccher, Lille de Sein et ses abords. Bull. A. G. F., a aril 1936 . oulven. C. R. Ac. Sc., t.188. 1929, 1 , p. 1261-1262. Gâvre. C.R.AC. Sc., 24 mars 1924 , p. 1084-1085.
(4) DANGEADD, Sur un ameurement de sables à nummulites près de Lorient. C. R. Somm. Soc. Geol.o 1925, p. 151. Géol. Minér. Bretagne, oct.-nov. 1936, p. 4-6. que les rives de l'anse du Pouldon, près de Pont-l'Abbé, recèlent des formations analogues, en particulier la rive $\mathbf{E}$. Là, depuis le niveau de la mer jusqu'à environ 25 mètres, se rencontrent de petits galets de quartz signalés sur la carte géologique, mais aussi des grès quartzites, des grès fins, souvent ferrugineux, roulés, des galets de silex et même de craie. Ces formations plongent sous les vases de l'anse, et on les retrouve, beaucoup moins abondantes il est vrai, sur la rive $W$.

Dans ces conditions, il est permis de supposer que la dépression entre les Glénan et la terre était formée dès l'éocène (1). Elle a dû de bonne heure attirer les rivières, ce qui expliquerait la douceur de lcur profil. La limite en est donnée en un point par les dreikanters.

Mais au cours des régressions quaternaires successives, le cours actuellement sous-marin de lodet a eu à subir les attaques de rivières plus jeunes, de direction S.-W.-N.-E. et remontant le lons du glacis des Glenan, de la Basse-Jaune et de line aux coutons. Cies thalwegs sous-marins ont un pronl beaucoup plus accusé. Bien que travaillant dans la bande de granulite de (1) Ce serait donc une cuvette ana logue à la mer du Morbihan. Des phénomènes
semblables ont dú se passer également plus à l'W, car dans la baie d’Audierne les mêmes galets se retrouvent dans les plages actuelies et anciennes.
ment and 


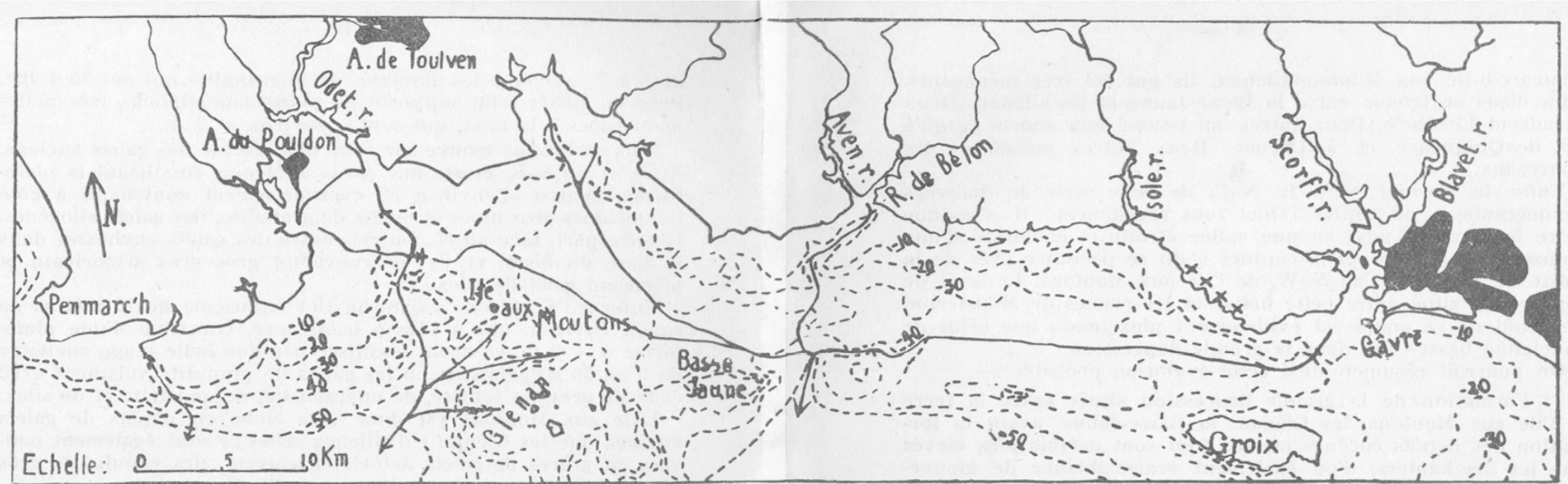

Fiti. 2. - Dépression. Les Glésan-Groix

dreikanters
éceene marin

isobathes

$\rightarrow \begin{aligned} & \text { principaux thalwegs sous-marin } \\ & \text { capture de l'Odet }\end{aligned}$

descendrait dans l'ensemble en pente assez douce. Autrement dit, les Glénan et le plateau de la Basse-Jaune seraient un bloc. bascule vers le S.-W.; sans en être sûr, on a quelques présomp tions de mouvements de ce genre sur d'autres points du littoral finisterien (1). L'abrupt de faille (ou de flexure) supposé s'atténue de plus en plus vers le N.-W.

Or, la ria de Quimper contient des dépôts marins éocènes (2) : grès analogues aux grès à sabals, calcaires, silex, On a trouvé aussi, rejetés par la mer et provenant des fonds voisins des calcaires écènes marins sur le rivage de la prescu'ile de Gâvre (3) : près de lá, entre les estuaires du Blavet et de rivière d'Etel, affleurent des sables glauconieux à nummuli tes (4). On a aussi signalé, sur le rivage entre l'Isole et le Scorff, des 4 dreikanters en grès éocenes contemporains des grès a Sabals 》 (5). Enfin, nous a

(1) GuILCHER, L'lle de Sein et ses abords. Bull. A, G. F., a arril 1936. Toulven. C. R. Ac. Sc., t.1 88 . 1929, Gâvre. C. R. Ac. Sc., 24 mars 1924, p. 1084-1085.
(4) DANGERD, Sur un ameurement de sables à nummulites près de Lorient. c. R. Somm. Soc. Gétol, 1925, pu. 151.
(5). PrinIProt, Quelques observations sur le littoral lorientais. C. R. Somm. Soc.
Géol. Miner. Bretagne, oct.-nov. 1936, p. 4-6. que les rives de l'anse du Pouldon, près de Pont-l'Abbé, recèlent des formations analogues, en particulier la rive $\mathbf{E}$. Là, depuis le niveau de la mer jusqu'à environ 25 mètres, se rencontrent de petits galets de quartz signalés sur la carte géologique, mais aussi des grès quartzites, des grès fins, souvent ferrugineux, roulés, des galets de silex et même de craie. Ces formations plongent sous les vases de l'anse, et on les retrouve, beaucoup moins abondantes il est vrai, sur la rive $W$.

Dans ces conditions, il est permis de supposer que la dépression entre les Glénan et la terre était formée dès l'éocène (1). Elle a dû de bonne heure attirer les rivières, ce qui expliquerait la douceur de lcur profil. La limite en est donnée en un point par les dreikanters.

Mais au cours des régressions quaternaires successives, le cours actuellement sous-marin de l'Odet a eu à subir les attaques de rivières plus jeunes, de direction S.-W.-N.-E. et remontant le long du glacis des Glenan, de la Basse-Jaune et de Thle aux Moutons. Cis thalwegs sous-mans ont un pronl beaucoup plus accusé. Bien que travaillant dans la bande de granulite d (1) Ce serait donc une cuvette analogue à la mer du Morbihan. Des phénomènes
semblables ont dú se passer également plus à l'W, car dans la baie d'Audierne les mèmes galets se retrouvent dans les plages actuelles et anciennes. 
Penmarc'h-ile aux Moutons-Glénan, ils ont été très menaçants. L'un d'eux se trouve entre la Basse-Jaune et les Glénan. Deux encadrent I,e Loc'h. Deux autres ont poussé leur source jusqu’à l'E. de Quignenec et à Drenec. Deux autres encadrent les Pourceaux.

Enfin le dernier, vers le N.-E. de cette série de thalwegs conquéránts, a dù capter l'Odet lout récemment. Il s'insinue entre les basses 'Tudy, en une vallée étroite et encaissće d'une quinzaine de mètres, et la capture a dû se produire près de la balise de Men Diou, au N.-W. de l'ile aux Moutons. Le seuil de capture est situé entre cette balise et les roches de Mousterlin. Le profil de ce cours est ividemment plus tendu que celui de l'ancienne basse-()det dans la grande dépression.

On pourrait résumer ainsi cette évolution probable:

1 . Formation de la grande dépression située ertre la terre et l'ile aux Moutons, les Glénan, la Basse-Jaune, avant la formation des dépôts éocènes marins (qui sont parfois plus élevés que les Ireikanters, d'où également vraisemblance de mouvements postérieurs) ;

$2^{\circ}$ Les rivières de la région se réunissent dans la dépression et franchissent l'abrupt de faille en un point unique;

$3^{\circ}$ Mouvements eustatiques quaternaires; création de niveaux d'abrasion marine vers 20 et 5 mètres sur la partie la plus élevée du bloc basculé; capture de l'Odet.

$4^{\circ}$ Transgression flandrienne et formation de la plate-forme actuelle.

III. Les genres de vie des iles de Glénan: Menhirs et allées couvertes ne manquent pas aux Glénan et à l'île aux Moutons. Ces terres étaient donc occupées dès le néolithique, de même que les îles du plateau molénais et l'île de Sein.

Il $y$ a une soixantaine d'années, la population était encore assez nombreuse pour que l'on se soit occupé d'entretenir un prêtre aux Glénan. Ce prêtre, l'abbé Thymeur, originaire de l'île de Sein, résidait au Loc'h où il officiait dans une petite chapelle, et les insulaires s'y réunissaient le dimanche.

Aujourd'hui, tout témoigne de la profonde décadence de ces terres isolées. Les Glénan sont de plus en plus abandonnées. Autrefois, le guetteur du sémaphore de Penfret était chargé de faire la classe aux enfants. On lui a ôté cette fonction, faute d'un nombre suffisant d'élèves. Lin des îlots de Quignenec était autrefois habité : il n'y reste plus qu'une maison en ruines.

L'activité des habitants se partage aujourd'hui entre deux genres de vie: la culture et le brûlage du goëmon; la pêche.

a) La culture et le brùlage du goëmon. Les iles appartiennent à de grands propriétaires : Saint-Nicolas, Guiautec, Penfret et Fort-Cigogne à une personne de Guilvinec, et le Loc'h à un pro- 
priétaire quimpérois. Ces gens louent certaines îles à des fermiers (Penfret est louée 2.300 francs) qui ont quelques vaches, un taureau, des chevaux, et cultivent du seigle, des pommes de terre, du foin et des choux fourragers dans les coins les moins pauvres. On trouve actuellement des fermes à Penfret, Drenec et Le Loc'h. Le brûlage du goëmon est aussi une occupation importante, comme dans les îles du plateau molénais. Mais malgré la décadence de ces dernières, la comparaison n'est pas à l'avantage des Glénan. Ici, point de ces « pigouilliers » ou occupants temporaires qui, de mars à octobre, viennent à côté des fermiers, dans les îles du plateau molénais, logeant dans de minuscules gourbis loués, avec un carré de dune pour le séchage du goëmon, 50 francs par an, et empanachent des fumées grises de leurs brûleries le Chenal du Four ; ici, le fermier fait seul de la « soude». C'est que les occupants temporaires des îles du Nord Finistère sont tous des «Pagan » de L'Abercorac'h ou Plouguerneau, et que les Glénan sont trop loin pour leurs minuscules bateaux. Les Pagan sont d'ailleurs ceux qui savent le mieux faire la soude. Le fermier du Loc'h en est un, et il produit plus de 20 tonnes par an contre 2 ou 3 pour celui de Penfret, originaire du Sud Finistère. Ces fermes sont d'ailleurs beaucoup moins importantes que dans l'autre archipel. Ce type d'économie est donc, aux Glénan, dans une décadence complète. On rencontre de nombreux trous de forme allongée, ayant servi à brûler le goëmon, et aujourd'hui abandonnés. Il y a bien quelques gens de la presqu'île de Penmarc'h qui viennent couper du goëmon aux Glénan : mais ils le brûlent en général sur le continent.

b) La pêche. La pêche, il est vrai, est moins en déclin. D’assez nombreux pêcheurs viennent aux Glénan passer la bonne saison. Ils sont logés de façon rudimentaire dans les casemates du Fort-Cigogne, et dans l'ancienne ferme, ainsi que les deux ou trois autres maisons de Saint-Nicolas. Aucune ilc n'est donc à la fois occupée par des pêcheurs et un fermier. Ces gens pêchent le homard et la langouste. C'est un type de pêche qui n'est pas en développement sur la côte Sud du Finistère : il s'agit d'ailleurs d'une petite pêche côtière, sans grande importance.

L'île aux Moutons n'est occupée que par un gardien de phare (il y a aussi deux gardiens de phare et deux guetteurs à Penfret); quant à Guiautec, Bananec, Brunec, Quignenec, etc., ce sont des illots déserts.

c) Le tourisme. Le tourisme aux Glénan s'est beaucoup développé récemment, et un hôtel s'est édifié à Saint-Nicolas. Il n'est pas rare, en été, de voir à Saint-Nicolas plusieurs grandes vedettes, venues surtout de Quimper. C'est la seule source de prospérité économique susceptible d'avenir aux Glénan, vivant par ailleurs d'une économie qui se meurt de plus en plus. 